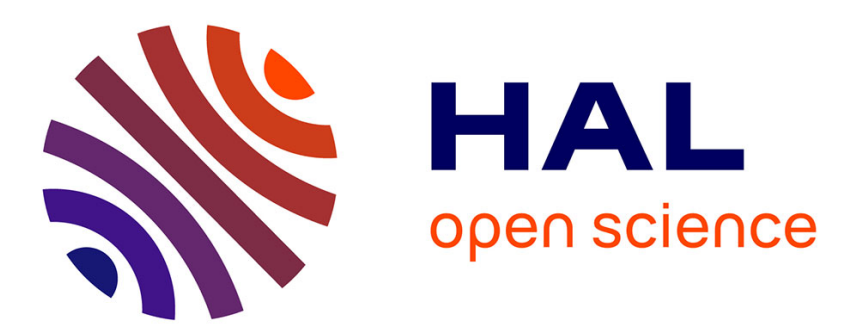

\title{
Evaluation of Channel Capacity of a 3D Curvilinear Metasurface in the $\mathrm{THz}$ band
}

\author{
Anna Maria Vegni, Valeria Loscri
}

\section{To cite this version:}

Anna Maria Vegni, Valeria Loscri. Evaluation of Channel Capacity of a 3D Curvilinear Metasurface in the $\mathrm{THz}$ band. IEEE Wireless Days, Jun 2021, Virtual (Paris), France. 10.1109/WD52248.2021.9508279 . ineris-03245803

\section{HAL Id: ineris-03245803}

\section{https://hal-ineris.archives-ouvertes.fr/ineris-03245803}

Submitted on 2 Jun 2021

HAL is a multi-disciplinary open access archive for the deposit and dissemination of scientific research documents, whether they are published or not. The documents may come from teaching and research institutions in France or abroad, or from public or private research centers.
L'archive ouverte pluridisciplinaire HAL, est destinée au dépôt et à la diffusion de documents scientifiques de niveau recherche, publiés ou non, émanant des établissements d'enseignement et de recherche français ou étrangers, des laboratoires publics ou privés. 


\section{Evaluation of Channel Capacity of a 3D Curvilinear Metasurface in the $\mathrm{THz}$ band}

\author{
Anna Maria Vegni \\ Dept. of Engineering \\ Roma Tre University \\ annamaria.vegni@uniroma3.it
}

\author{
Valeria Loscrí \\ FUN Research-lab \\ INRIA Lille-Nord Europe \\ valeria.loscri@inria.fr
}

\begin{abstract}
Metasurface structures (MSs) are able to control an impinging electromagnetic wave, in terms of amplitude and phase. Based on the prominent results of a specific curvilinear MS [1], designed and described by its equivalent nonhomogeneous Transmission Line (TL) model, in this paper we are motivated to investigate the achievable channel capacity bounds of the structure in the $\mathrm{THz}$ band.

Through its equivalent circuit model, we are able to derive the attenuation behavior of the proposed MS, which is dependent not only on the geometric features of the MS, but also on its TL components. We observe a MS geometry- and frequencydependence, which reflect also on the channel capacity upper bounds. Specifically, by varying the input power profile of an impinging electromagnetic wave, we can achieve different trends of the channel capacity. Best performance are reached assuming input femtosecond Gaussian-shaped power pulses, that allow to achieve Pbps channel capacity upper bounds.
\end{abstract}

Index Terms-Metasurfaces, channel capacity, attenuation, power profiles.

\section{INTRODUCTION}

In the last few years, research activities on beyond $5 \mathrm{G}$ have been started [2] with the main purpose of supporting enhanced mobile broadband (eMBB), massive communications [3], etc. Wireless communications in millimeter wave bands (i.e., above $20 \mathrm{GHz}$ and up to $300 \mathrm{GHz}$ ) is considered as a key enabler technology for the next generation of wireless systems. Indeed, this technology is expected to allow high data rate thanks to the huge available bandwidth. In [4], the authors discuss the "trade-offs" between scenario requirements and current silicon technologies limits, in order to push the next generation of wireless communication systems.

Different technologies and materials have been used to achieve this purpose, such as interferometers [5], miniaturized waveguides [6], gratings [7], [8], and the most recent 3D metamaterials [9], [10] i.e., artificial material that shows a frequency-dependent cross-coupling between the electric and magnetic fields. These materials show interesting advantages, but at the same time they need relatively large physical dimensions.

Among the various new revolutionary technologies proposed for the achievement of these objectives are metasurface structures (MSs), that present intriguing features not existing in nature. In the recent past, MSs have attracted attention for different interesting applications such as radar [11], holograms [12], and imaging [13]. Different MS configurations can be realized to work at different frequency ranges i.e., microwave, mm-waves, TeraHertz (THz), infrared and optics. The great versatility of these structures represents their main advantage since they can be used to control their response through modeling, design and manufacturing [14] or via software [15] for integration and miniaturization in existing platforms. On the other side, the exploitation of MSs in telecommunication is still at its embryonal stage, but it is starting arising more and more interest in the research community. Indeed, the capacity of manipulating electromagnetic (EM) waves is of paramount and great potential in wireless communications, as it is expected to easily achieve new performance limits.

Leveraging on the above motivations, in this paper we exploit a previously proposed approach [1] to practically realize 2D MSs, with properties not existing in nature, and investigate the benefits that can arise in terms of achievable channel capacity. The proposed technique is based on the use of the equivalent circuit-model theory, and is able to link the metasurface properties (i.e., amplitude and phase of an impinging wave) with its physical characteristics (i.e., inclusions' dimensions and spatial periodicity). The ability to control the EM waves is then expected to achieve interesting channel capacity limits in a metasurface medium.

In this paper, we study the behavior of the attenuation achieved in the proposed curvilinear MS. Then, by assuming different input power allocation schemes, we can derive variable channel capacity bounds. Specifically, we will focus on $\mathrm{THz}$ band spectrum and derive the channel capacity expression, both in respect of $(i)$ the characteristic parameters of the MS's TL model, (ii) the metasurface length, and (iii) the wave-number of an impinging signal.

This paper is organized as follows. In Section II we report the main related works about the use of MS for communication purpose, with particular emphasis to the channel capacity limits that can be achieved. Section III briefly describes the modeling and design of a particular metasurface structure, with specific geometrical and physical features, as previously introduced in [1]. We remind the mathematical description of the wave-structure interaction by means of the metasurface impedance, which relates the electromagnetic field components with the structure constitutive parameters. The expression of the metasurface impedance is also exploited in 
the formula of the attenuation, depending on both frequency and metasurface length. More details are reported in [1]. In Section IV, we derive the expression of channel capacity of a metasurface-based medium, which is strictly linked to the metasurface impedance. We provide the analysis of the metasurface channel capacity by varying different power profiles as input, and for each of them we investigate the capacity bounds that can be achieved. Section V presents numerical results that assess how the metasurface structure can achieve different channel capacity limits, based on specific input power profiles. It can be observed a dynamic behavior of the channel capacity depending on both $(i)$ the length of the metasurface, (ii) the wave-number, and (iii) the nature of the MS impedance. This allows the opportune tuning of the geometrical and physical features of the metasurface, as well as the working frequency, in order to obtain the full control of the transmitted signal and reach channel capacity upper bounds. Finally, conclusions are drawn at the end of the paper.

\section{RELATED WORKS}

Metasurfaces are artificial structures characterized with exotic EM features, not available in nature. Parameters such as wave amplitude, phase, and polarization can be effectively manipulated by the means of opportunistic discontinuities on the interface [16]. Metasurfaces are used with distinct phase profiles for orthogonal polarization states, which can be used as different optical components with various incident polarizations [17]. It follows that the arising interest of metasurfaces in the telecommunication context is justified by the rapid development of wireless networks systems demanding an increasing information capacity.

In order to meet this requirement, Kruk et al. in [18] employ the metasurface for both mode modulation and mode multiplexing for free-space optical communications. They focus on light modulation and more specifically, they consider the four dimensions that need to be regarded in order to enhance the information capacity i.e., time, wavelength, polarization and quadratures (amplitude and phase modulation), by arguing that a fifth dimension should be regarded in respect of the electromagnetic fields, namely the space. They consider metasurfaces as a key enabler way to realize miniaturization and integration of mode multiplexers and demultiplexers.

Another example about how MS can be employed from a telecommunication perspective is in [19], where the information encoding capacity and the increasing level of security are realized with multiple independent channels based on optical MSs. In [20], Wu et al. provide a quantitative framework that characterizes the MS capabilities in terms of information processing. The proposed framework is helpful for achieving a better insight of metasurfaces from an information theory point of view. In [21], Momeni et al. propose a new type of coding MS, based on graphene and working at $\mathrm{THz}$ frequencies. Inspired by an information-theory approach, the authors consider effective coding patterns in order to improve the far-field information and the channel capacity. Finally, another approach for enhancing the information capacity of metasurfaces is based on controlling spin-to-orbital angular momentum (OAM) [22].

Leveraging on the effective features of MSs, in this paper we aim to use a particular curvilinear MS in order to enhance communication performance, expressed in terms of channel capacity upper bounds. This can be achieved by accordingly choosing a power allocation scheme that better reaches performance enhancement. As known, an efficient resource allocation is of paramount in communication networks and can have a significant impact on system and communication performance.

Since for the general communication systems, besides the path loss effect and the noise, communication capabilities are strictly correlated to the distribution of power transmission, different power allocation schemes can result in different Signal-to-Noise Ratio (SNR) and information rate. In the simplest case, the power is uniformly distributed on the entire band (i.e., flat allocation scheme), while another approach consists in modeling the transmitted signal with an $n$-th derivative of a Gaussian shape pulse. In literature, there have been different power allocation solutions proposed to improve the performance of the system in terms of robustness, reliability, resilience, effectiveness, etc. Among the different schemes, Deng and Haimovich [23] propose a cooperative scheme among relay nodes. A power allocation scheme based on optimally allocation of sub-carriers by water-filling has been proposed in [24], by considering a constant rate power allocation. In [25], Liu et al. investigate the convergence speed of water-filling power allocation. Finally, an optimal power allocation scheme for relayed transmissions over Rayleighfading channels has been proposed in [26].

Based on the considerations regarding both $(i)$ different power allocation schemes and (ii) the fact that metasurfaces are gaining more and more interest in the communication research community, we address on a specific curvilinear MS [1] and investigate both the attenuation profile and the achievable channel capacity upper bounds for the proposed MS, by accordingly allocating input power profiles. To the best of our knowledge, these two aspects are both considered for the first time in the research community. Specifically, the main objectives of this paper can be enlisted as follows:

- The attenuation profile can be accordingly modeled by means of (i) wave-number, (ii) MS length and (iii) MS impedance;

- Different power allocation schemes i.e., (i) flat and (ii) pulse-based allocation schemes, will be considered and applied to a specific metasurface configuration working at the $\mathrm{THz}$ frequencies;

- The impact of the different input power allocation schemes will be evaluated by considering the reachable information capacity upper bounds.

\section{Metasurface Modeling And Design}

This section reminds the modeling and design of a particular curvilinear MS, as previously detailed in [1]. The proposed curvilinear metasurface is depicted in Fig. $1(a)$. It is formed by 


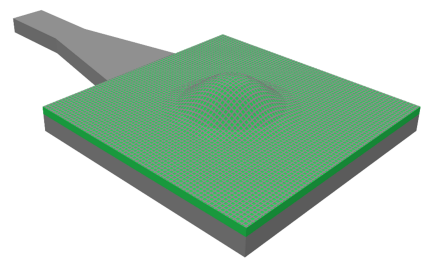

(a)

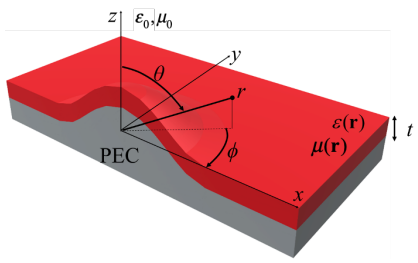

(b)
Fig. 1. Proposed metasurface design [1], observed from (a) perspective view, where the 3D curvilinear metasurface (green) is deposited on a grounded dielectric slab (grey), and (b) side view, where the equivalent non-homogeneous permittivity model of the metasurface structure i.e., $\varepsilon(\mathbf{r})$ is expressed in spherical coordinate system i.e., $(r, \theta, \phi)$.

(metallic/dielectric) patches, printed on a grounded dielectric substrate with relative permittivity $\varepsilon_{s l a b}$ and magnetic permeability $\mu_{s l a b}=\mu_{0}$, being $\mu_{0}$ as the magnetic permeability of free space. The impinging wave presents an incident angle $\theta$. The top layer is air with permittivity $\varepsilon_{0}$ and permeability $\mu_{0}$.

The proposed structure can be considered as a slab with nonhomogeneous constitutive parameters such as electric permittivity $\varepsilon(\mathbf{r})$ and/or magnetic permeability $\mu(\mathbf{r})$, function of the position vector $\mathbf{r}$, as also shown in Fig. $1(b)$. Let us consider the structure has thickness $t[\mathrm{~m}]$, and is electrically nonhomogenous $\varepsilon(\mathbf{r})$ and magnetically homogeneous $\mu_{r}=\mu_{0}$. According to Maxwells' and Heltmotz equations, the electric $\mathbf{E}$ and magnetic $\mathbf{H}$ vector equations are expressed respectively as:

$$
\nabla^{2} \mathbf{E}+\nabla[\mathbf{E} \bullet \nabla \log \varepsilon(\mathbf{r})]+\omega^{2} \mu \varepsilon(\mathbf{r}) \mathbf{E}=0,
$$

and

$$
\nabla^{2} \mathbf{H}+\nabla \log \varepsilon(\mathbf{r}) \times(\nabla \times \mathbf{H})+\omega^{2} \mu \varepsilon(\mathbf{r}) \mathbf{H}=0 .
$$

As previously introduced in [1], the proposed metasurface structure has been described by its equivalent nonhomogeneous TL model along the $(r, \theta, \phi)$ directions.

For each TL, the related power transfer function can be evaluated. In case of radial direction $r$, let us assume the following expression for the voltage along $r$ i.e.,

$$
V(r)=a_{1} e^{-\alpha r}+a_{2} e^{\alpha r},
$$

where the first term represents the propagating wave and the second term the reflected one. Specifically, $a_{1}$ and $a_{2}$ are the amplitudes of the waves and $\alpha$ is the complex wavenumber. Furthermore, we consider that voltage and current at the beginning and end of the line of length $d[\mathrm{~m}]$ are, respectively $\left(V_{0}, I_{0}\right)$ and $\left(V_{d}, I_{d}\right)$. It follows that the out-in power transfer function $H$ can be derived as

$$
H=\frac{P_{\text {out }}}{P_{\text {in }}}=\frac{4 e^{-\left(\alpha+\alpha^{*}\right) r}}{Z_{r}(r)}\left[\frac{|\zeta|^{2}}{(1+\zeta)^{2}-(1-\zeta)^{2} e^{-4 r \alpha}}\right],
$$

where $Z_{r}$ is the non-homogeneous impedance profile for the metasurface along the radial direction $r$, the symbol * represents the conjugated operator, and $\zeta$ represents the ratio between the load impedance $Z_{\text {load }}$ and $Z_{r}(r)$, i.e.,

$$
\zeta=-\frac{Z_{\text {load }}}{Z_{r}(r)} .
$$

Similar expressions can be derived for the TL along the other directions. Notice that $Z_{\text {load }}$ can be expressed through its inductive and/or capacitive nature, i.e.,

$$
Z_{\text {load }}=R+j \omega L+\frac{1}{j \omega C},
$$

with $\omega=2 \pi f$ as the frequency. As Eq. (6) can show a frequency-dependent behavior, we expect that also the power transfer function out-in $H$ depends on the frequency, in case that $Z_{\text {load }}$ has a capacitive and/or inductive nature.

Finally, from Eq. (4), we can easily compute the attenuation $[\mathrm{dB}]$ as a function of the metasurface length $r[\mathrm{~m}]$ i.e.,

$$
L(r)=-10 \log (H) .
$$

It follows that also the attenuation is dependent on the frequency, as well as the MS geometric features and the corresponding TL model components. This result can be exploited to achieve other important advances in metasurface-based communications. Indeed, we expect to observe a frequencyand geometry-dependent behavior of channel capacity, reaching upper limits under different assumptions for power spectral density (p.s.d.) within the whole band of interest i.e., $(0.1-10)$ THz.

\section{Metasurface-Based Channel Capacity ANALYSIS}

In this section, we investigate the capacity behavior of a metasurface, as designed in Section III, working in the $\mathrm{THz}$ band.

Let us consider the received signal in a metasurface channel as a sum of several contributions, each one transmitted in the $i$-th sub-band, showing a narrow behavior and a flat-band response i.e.,

$$
\sum_{i=1}^{N_{B}} P_{i} \leq P_{T O T}
$$

where $N_{B}$ is the total number of sub-bands that compose the total bandwidth $B[\mathrm{~Hz}], P_{i}[\mathrm{dBm}]$ is the transmission power in the $i$-th sub-band, and $P_{T O T}$ is the total transmitted power in the $\mathrm{THz}$ band, i.e., $B=[0.1,10] \mathrm{THz}$. Then, the overall capacity $\mathcal{C}$ can be defined as the sum of the single capacities $C_{i}$ in each of $N_{B}$ sub-bands i.e.,

$$
\mathcal{C}=\sum_{i=1}^{N_{B}} C_{i}=\sum_{i=1}^{N_{B}} \Delta f_{i} \log \left(1+\frac{\left|h_{i}\right|^{2} P_{i}}{\Delta f_{i} S_{N}\left(f_{i}\right)}\right),
$$

where $h_{i}$ is the $i$-th power transfer function in the $i$-th subband, whose expression has been previously introduced in Eq. (4), $S_{N}$ is the power spectral density of the additive white Gaussian noise and $\Delta f_{i}$ is the sub-band range among to consecutive sub-bands i.e., $\Delta f_{i}=f_{i+1}-f_{i}$, with $i=$ $1,2, \ldots, N_{B}$. Specifically, we assume a gap of $10 \mathrm{GHz}$ among 


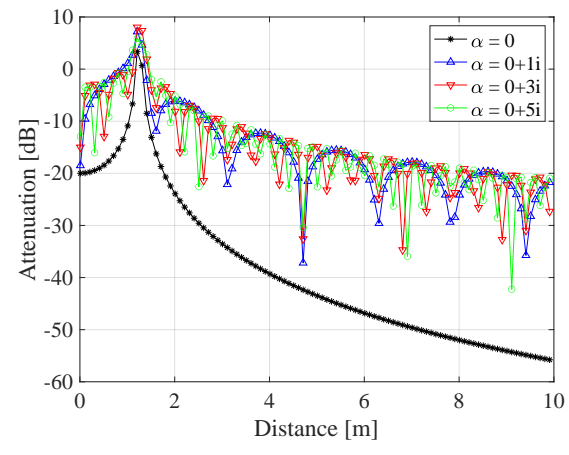

(a)

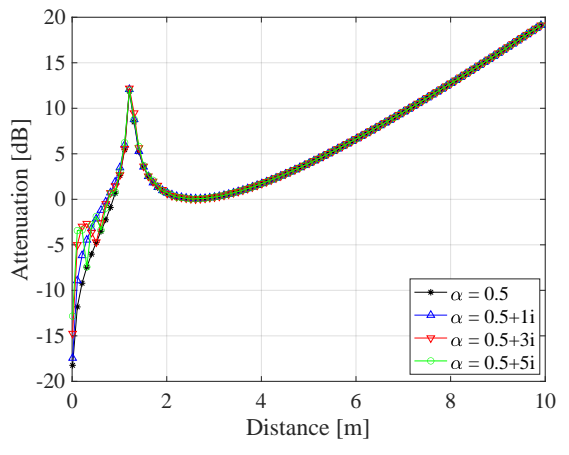

(b)

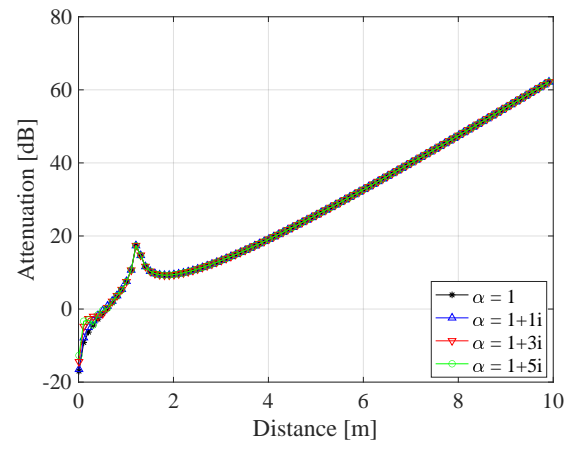

(c)

Fig. 2. Attenuation $[\mathrm{dB}]$ behavior versus the distance in case of $(a) \operatorname{Re}[\alpha]=0,(b) \operatorname{Re}[\alpha]=0.5$ and $(c) \operatorname{Re}[\alpha]=1$.

two consecutive sub-bands and we obtain $N_{B}=990$ subbands in the whole $\mathrm{THz}$ band.

It can be easily noticed that the channel capacity expression in Eq. (9) is expected to be dependent directly on the metasurface parameters, such as $Z_{0}$ and $Z_{\text {load }}$, as appearing in Eq. (4). Furthermore, the metasurface channel capacity is expected to be geometry-dependent through the expression of $\left|h_{i}\right|$, as well as depends on the specific input power profile adopted in the $i$-th sub-band i.e., $P_{i}$. Notice that also the power profile can assume a frequency-dependent behavior, and then different values of channel capacity per sub-band depend on a given power allocation scheme adopted in the $i$-th sub-band.

Regarding the transmission strategies, we consider two approaches that differently distribute the input power in the frequency domain i.e., in the $\mathrm{THz}$ band. The simplest case of power allocation scheme considers that the power level in each sub-band is equally distributed over the entire operative band $B$ [THz], namely flat power profile, i.e.,

$$
P_{i}=P_{\text {flat }}(f)= \begin{cases}P_{0}, & \forall f \in B \\ 0, & \text { otherwise }\end{cases}
$$

where $P_{0}[\mathrm{dBm}]$ is the constant power level in each sub-band.

Finally, the second approach for power allocation is related to the possibility of transmitting very short pulses in order to hundred of femtoseconds. Typically, in $\mathrm{THz}$ band, these pulses are modeled following a Gaussian distribution, i.e., $\mathcal{N}\left(\mu, \sigma^{2}\right)$, with $\mu$ [s] as the mean value of the distribution and $\sigma$ [s] as the standard deviation of the Gaussian pulse. The power spectral distribution (p.s.d) of the $n$-order time derivate of a femtosecond-long pulse is also Gaussian shaped, and the power level in the $i$-th sub-band shows the following profile i.e.,

$$
P_{i}=P_{p u l s e}^{(n)}(f)=\mathcal{A}^{2}(2 \pi f)^{2 n} \exp \left[-(2 \pi \sigma f)^{2}\right]
$$

where $\mathcal{A}$ is a normalizing constant useful to adjust the pulse total energy, which can be computed as

$$
\mathcal{A}^{2}=\frac{P_{i}}{\int_{f_{\min }}^{f_{\max }} P_{\text {pulse }} d f},
$$

where $f_{\min }$ and $f_{\max }$ are the lower and the higher operative frequencies that indicate the overall bandwidth $B[\mathrm{~Hz}]$, respectively. Specifically, in case of $\mathrm{THz}$ band, $f_{\min }=0.1$ and $f_{\max }=10 \mathrm{THz}$. The expression of power in Eq. (11) allows to identify the pulse-based power allocation scheme.

\section{Attenuation and Channel Capacity Limits}

The following results represent the behavior of channel capacity per sub-band in a metasurface medium, designed according to the proposed unit cell model, as introduced in [1]. All the results have been obtained in Matlab environment. We are interested in the analysis of both the attenuation and the channel capacity upper bounds along the radial direction and for different values of complex wavenumber $\alpha$ i.e., $\operatorname{Re}[\alpha]=[0,0.5,1]$ and $\operatorname{Im}[\alpha]=[0,0.5,1,4]$. The behavior of the attenuation at the output of the metasurface allows to understand how the emitted signal can be controlled, and then how effective the performance of the channel are.

We present the impact of the parameter $\alpha$ on the signal propagation in respect of the radial direction, in case of no frequency dependence, assuming $Z_{\text {load }}=R=100 \Omega$, in order to highlight how the geometry features of the metasurface affect the performance. Fig. 2 describes the attenuation profiles for $r=10[\mathrm{~m}]$, in case of variable $\alpha$ i.e., $\operatorname{Re}[\alpha]=[0,0.5,1]$ and $\operatorname{Im}[\alpha]=[0,1,3,5]$. In case of $\operatorname{Re}[\alpha]=0$ as depicted in Fig. $2(a)$, it is interesting to observe peaks of attenuation around $\approx 10 \mathrm{~dB}$ for $r \approx 1.2 \mathrm{~m}$, i.e., in such a specific length the propagated signal shows the highest attenuation. We evince the powerful feature of metasurface that allows to fully control the impinging signal. The peak of attenuation is followed by a decreasing slope for $r>1.2 \mathrm{~m}$, with a very dynamic behavior in case of $\alpha=\operatorname{Im}[\alpha]$, with peaks and fluctuations of the attenuation caused by the inhomogeneity of the medium, while the trend is smoother and strongly decreasing with the radial direction in case of $\operatorname{Im}[\alpha]=0$.

Notice that a decreasing behavior of the attenuation along the distance is unusual w.r.t typical attenuation trends, which show increasing behavior with the channel length. This aspect is due to $\operatorname{Re}[\alpha]=0$ that allows to generate a gain (i.e., amplification) of the output power for higher distances. Once again, 


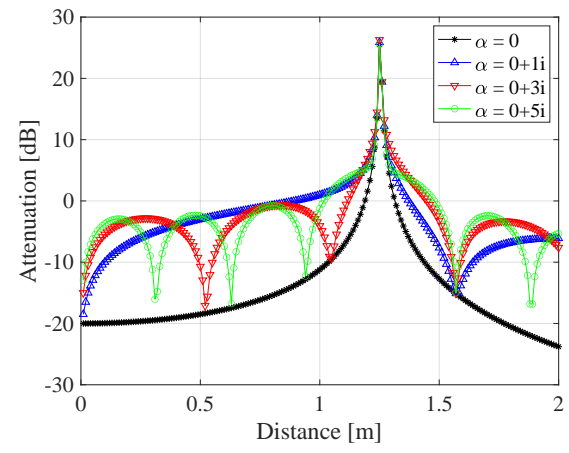

(a)

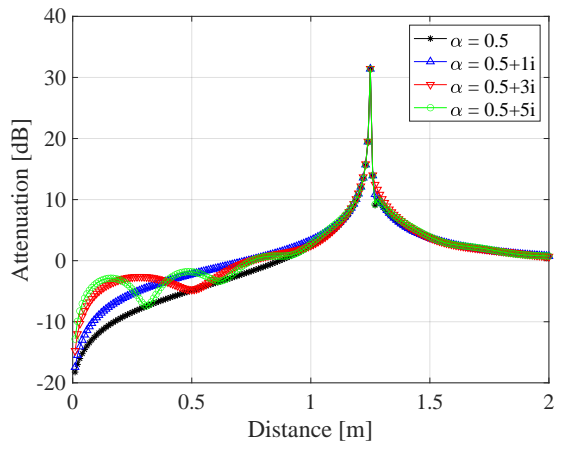

(b)

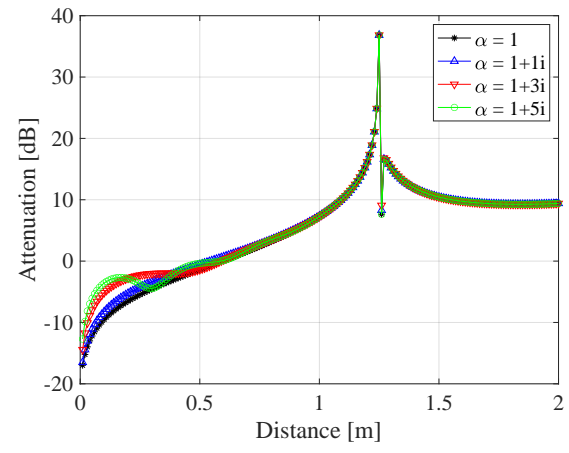

(c)

Fig. 3. Attenuation $[\mathrm{dB}]$ behavior versus the distance in case of $(a) \operatorname{Re}[\alpha]=0,(b) \operatorname{Re}[\alpha]=0.5$ and $(c) \operatorname{Re}[\alpha]=1$.

this result represents the powerful feature of metasurfaces that can control EM waves.

Peaks of attenuations can be observed also in case of increasing $\operatorname{Re}[\alpha]$, as in Fig. $2(b)$ and $(c)$, where for short length of the propagation medium i.e., $r \leq 1.2[\mathrm{~m}]$, there is a decreasing behavior of the attenuation, followed by a smoother and very dynamic trend for increasing distances (i.e., longer the channel, higher the attenuation loss). We observe that for higher $\operatorname{Re}[\alpha]$, fluctuations and peaks diminish in favor of a smoother trend. It looks like increasing real part of wavenumber $\alpha$ reduces peaks and fluctuations in favor of an increasing and smoother attenuation trend. Finally, for $\operatorname{Re}[\alpha]>0$, maximum values of the attenuation are $\approx 30 \mathrm{~dB}$ at distance $r=10 \mathrm{~m}$. As a result, increasing $\operatorname{Re}[\alpha]$ provides an increase of attenuation with smooth trend.

Such behaviors can be better observed in Fig. 3 that shows a zoom of the attenuation trend for $r \leq 2 \mathrm{~m}$, and for different wavenumbers $\alpha$. Again, peaks and fluctuations disappear in flavor of a smooth behavior in case of $\alpha=0$ (see black curve in Fig. $3(a)$ ), showing very low values of attenuation, thus reflecting an amplification of the impinging signal. Fluctuations of attenuations are observed for increasing $\operatorname{Im}[\alpha]$ (see blue, red and green lines). Differently, in Fig. $3(b)$ and $(c)$, for $\operatorname{Re}[\alpha]>0$, the attenuation peaks are around $\approx 30[\mathrm{~dB}]$ and $\approx 40[\mathrm{~dB}]$, respectively, and fluctuations are strongly reduced.

To summarize, we can evince the following behaviors of the attenuation of a signal impinging on the proposed curvilinear metasurface structure in case of $Z_{\text {load }}=R$, i.e.:

- As expected, the attenuation increases with the metasurface length, only when $\operatorname{Re}[\alpha]>0$, with a resonance peak around $r \approx 1.2 \mathrm{~m}$;

- For $\operatorname{Re}[\alpha]=0$, the attenuation trend decreases with the metasurface length, due to no losses introduced by the wave-number;

- Increasing $\operatorname{Re}[\alpha]$ provides enhanced attenuation, while increasing $\operatorname{Im}[\alpha]$ highlights peaks and fluctuations of the attenuation;

- For $\operatorname{Re}[\alpha]>0$, the resonance peak occurs at $r \approx 1.2 \mathrm{~m}$ and is followed by an increasing slope;

- When $\alpha=\operatorname{Im}[\alpha] \neq 0$, fluctuations present a periodic trend that decreases with the metasurface length.

After depicting the attenuation trend of an impinging signal on a metasurface channel, we are able to investigate the channel capacity upper bounds for different power allocation schemes. In Fig. 4 we report the values of channel capacity achieved for $r=10 \mathrm{~m}$, in case of a resistive load impedance i.e., $Z_{\text {load }}=R$, and a flat input power profile i.e., $P_{0}=$ $46 \mathrm{dBm}$. Again, performance have been obtained in case of variable complex wavenumber $\alpha$. In Fig. $4(a)$, it can be observed $(i)$ a fluctuation trend along the metasurface structure when $\alpha=\operatorname{Im}[\alpha]$ that is, the imaginary part of wavenumber prevents the smooth behavior, and (ii) a decreasing trend for short metasurface size i.e., $r \leq 1.2 \mathrm{~m}$. Such features are also reflected in the attenuation trend, as in Fig. 2. The increasing capacity trend is due to the absence of losses in the metasurface, as introduced by $\operatorname{Re}[\alpha]=0$. In this case, we observe high values of capacity, reaching $\approx$ Pbps order (see black curve in Fig. $4(a)$ ). In Fig. $4(b)$ and (c), when increasing the real part of $\alpha$, channel capacity degrades with increasing metasurface length, with values of the order of Mbps for very short lengths. Indeed, we observe a severe drop of channel capacity around $r \approx 1.2 \mathrm{~m}$, followed by a smooth increase, and then again a decreasing slope for higher metasurface lengths. This aspect is due to the increase of the real part of $\alpha$ that provides higher attenuation to the impinging signal.

As noticed in Fig. 4, channel capacity performance reach $\approx$ Gbps and $\approx$ Pbps order for $\operatorname{Re}[\alpha]=0$, while for increasing $\operatorname{Re}[\alpha]$, the channel capacity bound is limited to Mbps order for very short metasurface lengths, as depicted in Fig. 4 (b) and $(c)$. It is also observed that for higher MS lengths, the channel capacity shows a severe decrease.

Differently, better performance are obtained in case transmission of Gaussian-based power pulses, assuming $\sigma=$ $[50,100,150]$ fs. Fig. 5 depicts the channel capacity upper bound for $r \leq 10 \mathrm{~m}$, obtained assuming a pulse-based input power profile, in case of different values of wave-number $\alpha$. Again, we assumed $Z_{\text {load }}=100 \Omega, \operatorname{Re}[\alpha]=[0,0.5,1]$ and $\operatorname{Im}[\alpha]=[1,5]$. Also, according to Eq. (11), performance are affected by the derivative order $n$ of the Gaussian pulse. Specifically, we distinguish channel capacity upper bounds 


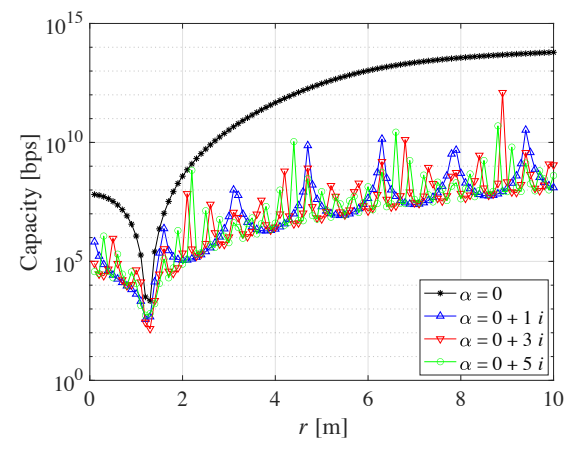

(a)

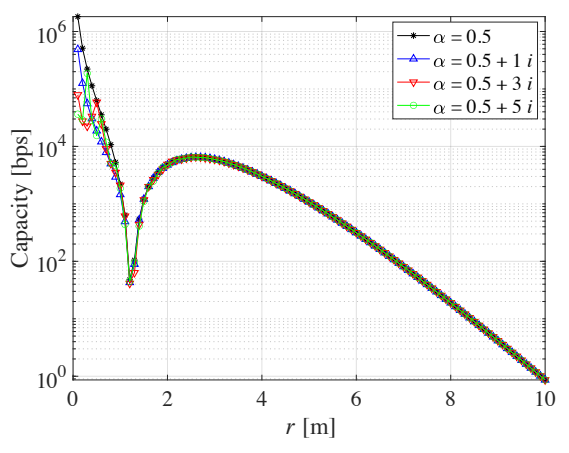

(b)

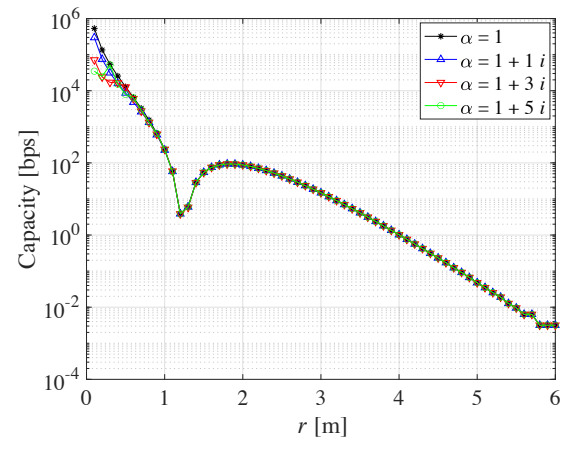

(c)

Fig. 4. Capacity [bps] behavior in case of flat power profile, versus the distance and for $Z_{\text {load }}=R=100 \Omega$, in case of $(a) \operatorname{Re}[\alpha]=0,(b) \operatorname{Re}[\alpha]=0.5$, and $(c) \operatorname{Re}[\alpha]=1$.

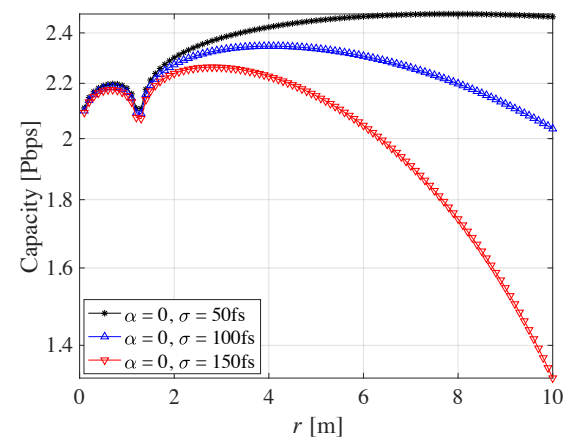

(a)

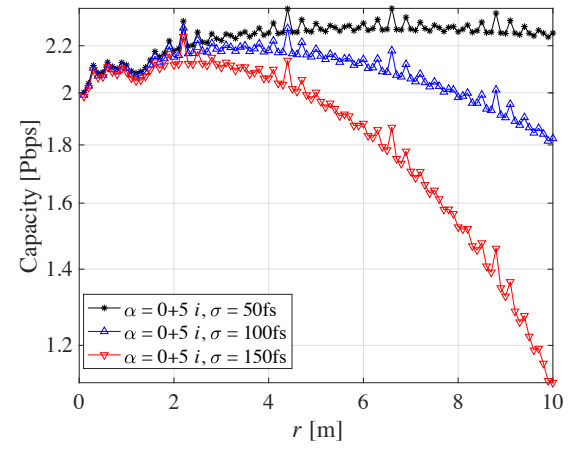

(d)

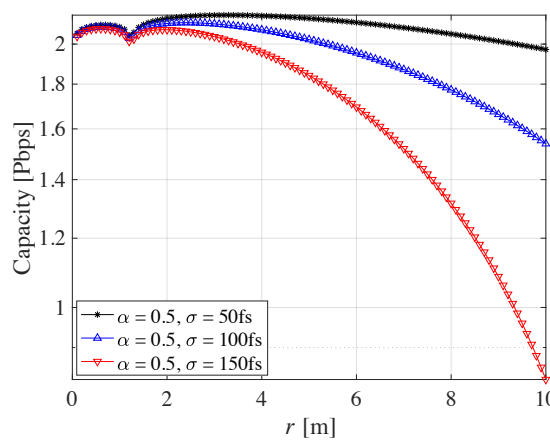

(b)

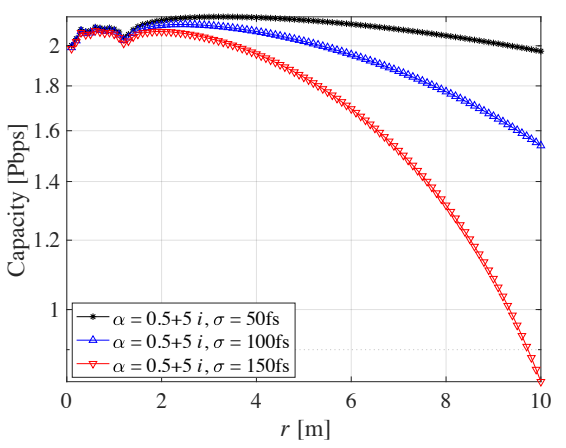

(e)

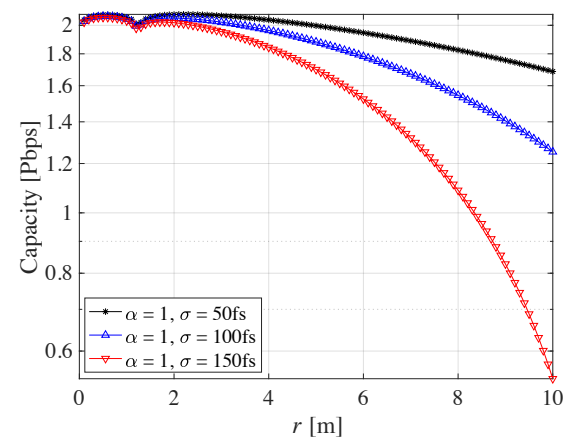

(c)

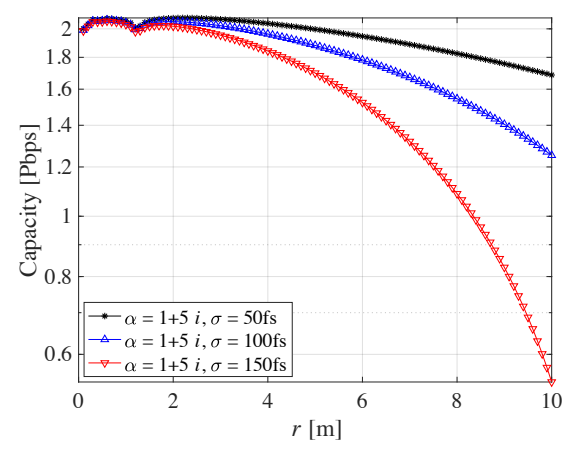

$(f)$

Fig. 5. Capacity [Pbps] behavior in case of pulse power profile, versus the distance, for $Z_{\text {load }}=R=100 \Omega$ and $n=1$, in case of Re $[\alpha]=[0,0.5,1]$ and $\operatorname{Im}[\alpha]=[0,5]$.

obtained for (i) low (i.e., $n=1$ ) and (ii) high derivative order (i.e., $n=6$ ), and results are reported in Fig. 5 and 6 , respectively. Firstly, both for low and high derivative order, we notice that the channel upper bounds are in the order to Pbps, that represents a very enhanced achievement w.r.t. the channel capacity results obtained in case of flat power profile. Of course, different ranges are obtained in case of low and high derivative number, so that the channel capacity upper bounds reach $\approx 2 \mathrm{Pbps}$ and $\approx 7 \mathrm{Pbps}$, as depicted in Fig. 5 and 6, respectively. More in detail, in Fig. 5 for $n=1$, the channel capacity upper bound starts around 2 Pbps for low metasurface length, with a resonance peak at $r=1.2 \mathrm{~m}$, as already observed for the attenuation trend in Fig. 3. Capacity bounds have been observed for different values of $\sigma$, so that short pulses i.e., $\sigma=50 \mathrm{fs}$, provide higher capacity values (see black curves in Fig. 5), with small decreases with the metasurface length in case of increasing $\operatorname{Re}[\alpha]$, as observed in Fig. $5(b)$ and $(c)$. Similar considerations apply for Fig. 5 $(e)$ and $(f)$, where we observe a channel capacity values of $\approx 2 \mathrm{Pbps}$ and $\approx 1.7 \mathrm{Pbps}$ at $r=10 \mathrm{~m}$, both in case of $\sigma=50 \mathrm{fs}$, respectively. On the other side, when a longer power pulse signal impinges the metasurface (i.e., $\sigma=150 \mathrm{fs}$ ), the channel capacity presents a very dynamic decreasing trend for higher metasurface lengths (see red curves in Fig. 5). Also, 


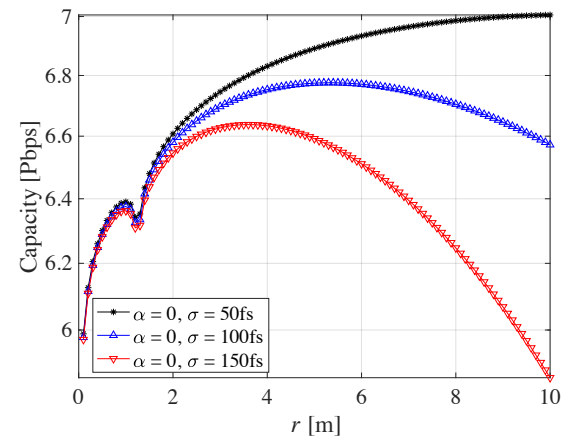

(a)

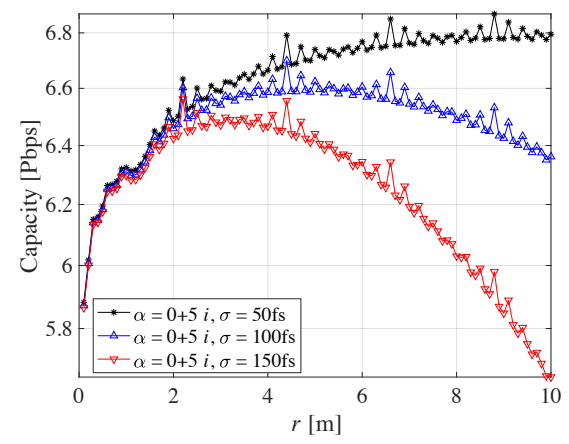

(d)

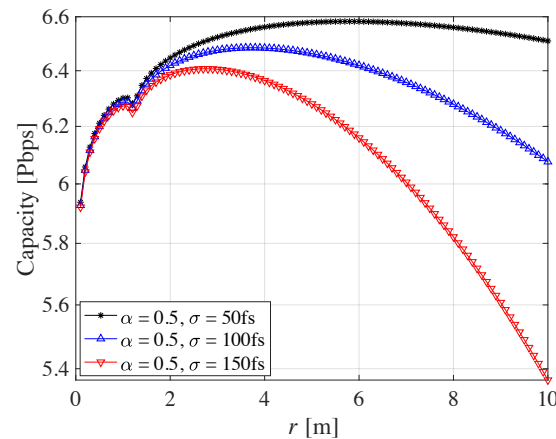

(b)

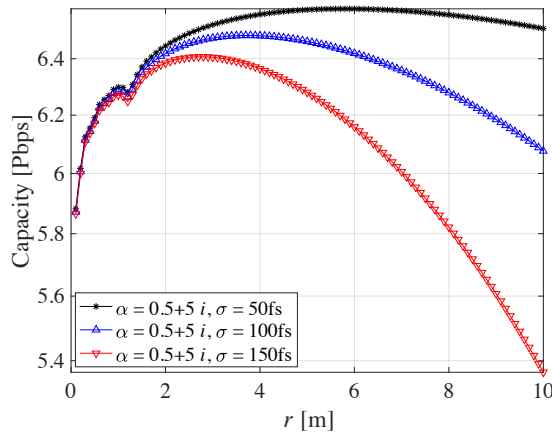

$(e)$

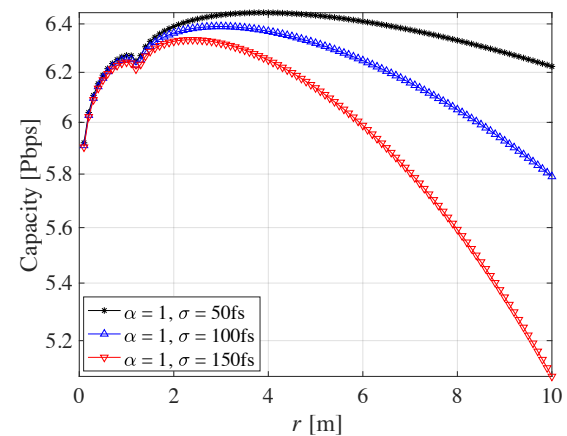

(c)

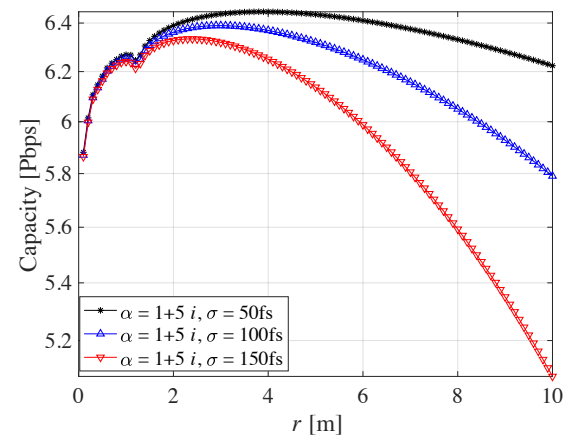

$(f)$

Fig. 6. Capacity [Pbps] behavior in case of pulse power profile, versus the distance, for $Z_{\text {load }}=R=100 \Omega$ and $n=6$, in case of $\operatorname{Re}[\alpha]=[0,0.5,1]$ and $\operatorname{Im}[\alpha]=[0,5]$.

increasing the real part of $\alpha$ provides a degradation of the channel capacity upper bounds for fixed metasurface lengths. Finally, as expected, when $\alpha=\operatorname{Im}[\alpha] \neq 0$, fluctuations of the channel capacity appear, as depicted in Fig. $5(d)$.

Similar considerations can be observed in Fig. 6 for high derivative order $n$, except a more dynamic trend of the channel capacity that increases faster with the metasurface length (see black curves). Also, the channel capacity values are higher than the case of low derivative order, around $\approx 6 \mathrm{Pbps}$. Finally, small decreases are noticed for increasing $\operatorname{Re}[\alpha]$ with increasing metasurface length.

\section{CONCLusions}

Metasurfaces are recently arising a lot of interest in telecommunication research community, due to their great versatility in the manipulation of electromagnetic wave. The potential of integrating a communication system with such a kind of structure is paramount, since the performance of the system could benefit of it by equipping for example offices walls or in general building walls with metasurface structures.

Based on these premises, in this work we have considered a curvilinear MS, presenting very intriguing properties in terms of electromagnetic wave manipulation. In the perspective of MS integration in a communication system, we have investigated the channel capacity upper bounds that can be achieved in the $\mathrm{THz}$ band. In particular, since power and resource allocation have been for a long time investigated in the communication network community showing their effectiveness, we have applied different power allocation mechanisms on our MS structure, and shown how these approaches may impact on the channel capacity performance. As a conclusion, we have observed higher performance in case of femtosecond Gaussian pulses of high derivative order, as compared to a flat power profile. Furthermore, the complex nature of wavenumber allows to accordingly achieve smoother or fluctuating channel capacity behaviors.

\section{REFERENCES}

[1] L. La Spada, V. Loscrí, and A. M. Vegni, "MetaSurface Structure Design and Channel Modelling for $\mathrm{THz}$ Band Communications," in IEEE INFOCOM 2019 - IEEE Conference on Computer Communications Workshops (INFOCOM WKSHPS), April 2019, pp. 708-713.

[2] K. David and H. Berndt, "6G Vision and Requirements: Is There Any Need for Beyond 5G?" IEEE Vehicular Technology Magazine, vol. 13, no. 3 , pp. $72-80,2018$.

[3] M. Shafi, A. F. Molisch, P. J. Smith, T. Haustein, P. Zhu, P. De Silva, F. Tufvesson, A. Benjebbour, and G. Wunder, "5G: A Tutorial Overview of Standards, Trials, Challenges, Deployment, and Practice," IEEE Journal on Selected Areas in Communications, vol. 35, no. 6, pp. 1201-1221, 2017.

[4] J. Doré, D. Belot, E. Mercier, S. Bicaïs, G. Gougeon, Y. Corre, B. Miscopein, D. Kténas, and E. C. Strinati, "Technology Roadmap for Beyond 5G Wireless Connectivity in D-band," in 2020 2nd $6 G$ Wireless Summit (6G SUMMIT), 2020, pp. 1-5.

[5] Z. Qi, N. Matsuda, K. Itoh, M. Murabayashi, and C. R. Lavers, "A Design for Improving the Sensitivity of a Mach-Zehnder Interferometer to Chemical and Biological Measurands," Sens. Actuators B, vol. 81, pp. $254-258,2002$.

[6] F. Dell'Olio and V. M. N. Passaro, "Optical sensing by optimized silicon slot waveguides," Optics Express, vol. 15, pp. 4977-4993, 2007. 
[7] G. Veldhuis, J. H. Berends, R. G. Heideman, and P. V. Lambeck, "An integrated optical Bragg reflector used as a chemo-optical sensor," Pure Appl. Opt., vol. 7, pp. 23-26, 1998.

[8] I. L. Y. Li, C. D. Giovampaola, and N. Engheta, "Waveguide metatronics: Lumped circuitry based on structural dispersion," Science Advances, vol. 12, no. 6, 2016.

[9] A. M. Vegni and V. Loscrí, "Analysis of the Chirality Effects on the Capacity of Wireless Communication Systems in the THz band," IEEE Transactions on Wireless Communications, vol. 16, no. 12, pp. 7848 7858, 2017.

[10] A. Taibi, A. Durant, V. Loscrí, A. M. Vegni, and L. L. Spada, "Controlling light by curvilinear metasurfaces," in Proceedings of the Sixth Annual ACM International Conference on Nanoscale Computing and Communication, ser. NANOCOM '19. New York, NY, USA: Association for Computing Machinery, 2019. [Online]. Available: https://doi.org/10.1145/3345312.3345484

[11] P. Su, Y. Zhao, S. Jia, W. Shi, and H. Wang, "A broadband random metasurface for Radar Cross Section reduction," in 2015 Asia-Pacific Microwave Conference (APMC), vol. 2, 2015, pp. 1-3.

[12] Q. Jiang, G. Jin, and L. Cao, "When Metasurface meets Hologram: Principle and Advances," Adv. Opt. Photon., vol. 11, no. 3, pp. 518-576, Sep 2019. [Online]. Available: http://aop.osa.org/abstract. cfm?URI=aop-11-3-518

[13] B. Lee and G. Lee, "Metasurfaces for Display and Imaging Applications," in 2019 International Conference on Optical MEMS and Nanophotonics (OMN), 2019, pp. 196-197.

[14] T. J. Yen, W. J. Padilla, N. Fang, D. C. Vier, D. R. Smith, J. B. Pendry, D. N. Basov, and X. Zhang, "Terahertz magnetic response from artificial materials," Science, vol. 303, pp. 1494-1496, 2004.

[15] C. Liaskos, S. Nie, A. Tsioliaridou, A. Pitsillides, S. Ioannidis, and I. Akyildiz, "A New Wireless Communication Paradigm through Software-controlled Metasurfaces," IEEE Communications Magazine, vol. 56, no. 9, pp. 162-169, 2018.

[16] N. Zhang, K. Chen, Y. Zheng, Q. Hu, K. Qu, J. Zhao, J. Wang, and Y. Feng, "Programmable coding metasurface for dual-band independent real-time beam control," IEEE Journal on Emerging and Selected Topics in Circuits and Systems, vol. 10, no. 1, pp. 20-28, 2020.

[17] L. Yan, W. Zhu, M. F. Karim, H. Cai, A. Y. Gu, Z. Shen, P. H. J. Chong, D. P. Tsai, D.-L. Kwong, C.-W. Qiu, and A. Q. Liu, "Arbitrary and Independent Polarization Control In Situ via a Single Metasurface," Advanced Optical Materials, vol. 6, no. 21, p. 1800728, 2018. [Online]. Available: https://onlinelibrary.wiley.com/doi/ abs/10.1002/adom.201800728

[18] S. Kruk, F. Ferreira, N. Mac Suibhne, C. Tsekrekos, I. Kravchenko, A. Ellis, D. Neshev, S. Turitsyn, and Y. Kivshar, "Transparent Dielectric Metasurfaces for Spatial Mode Multiplexing," Laser \& Photonics Reviews, vol. 12, no. 8, p. 1800031, 2018. [Online]. Available: https://onlinelibrary.wiley.com/doi/abs/10.1002/lpor.201800031

[19] F. Dong and W. Chu, "Multichannel-Independent Information Encoding with Optical Metasurfaces," Advanced Materials, vol. 31, no. 45, p. 1804921, 2019. [Online]. Available: https://onlinelibrary.wiley.com/doi/ abs/10.1002/adma.201804921

[20] H. Wu, G. D. Bai, S. Liu, L. Li, X. Wan, Q. Cheng, and T. J. Cui, "Information theory of metasurfaces," National Science Review, vol. 7, no. 3, pp. 561-571, 11 2019. [Online]. Available: https://doi.org/10.1093/nsr/nwz195

[21] A. Momeni, K. Rouhi, H. Rajabalipanah, and A. Abdolali, "An Information Theory-Inspired Strategy for Design of Re-programmable Encrypted Graphene-based Coding Metasurfaces at Terahertz Frequencies," Scientific Reports, vol. 8, 2018.

[22] G. Ding, K. Chen, X. Luo, J. Zhao, T. Jiang, and Y. Feng, "Dual-Helicity Decoupled Coding Metasurface for Independent Spin-to-Orbital Angular Momentum Conversion," Physical review applied, vol. 11, p. 044043, 2019.

[23] Xitirnin Deng and A. M. Haimovich, "Power allocation for cooperative relaying in wireless networks," IEEE Communications Letters, vol. 9, no. 11, pp. 994-996, 2005.

[24] C. Liu, A. Schmeink, and R. Mathar, "Constant-rate power allocation under constraint on average ber in adaptive ofdm systems," in 2010 IEEE International Conference on Communications, 2010, pp. 1-5.

[25] H. Moon, "Waterfilling Power Allocation at High SNR Regimes," IEEE Transactions on Communications, vol. 59, no. 3, pp. 708-715, 2011.
[26] M. O. Hasna and M. . Alouini, "Optimal power allocation for relayed transmissions over Rayleigh-fading channels," IEEE Transactions on Wireless Communications, vol. 3, no. 6, pp. 1999-2004, 2004. 